Cramps in the affected leg are quite common and persistent although sciatic pain may have disappeared completely.

A small proportion of patients develop a true recurrent prolapse (prolapse from the same disc) months to years later. This varies in individual statistics, but is probably in the neighbourhood of 4 per cent. to 5 per cent. These recurrent attacks of sciatica, due to a further protrusion, are dealt with in the same way as the primary attacks, except that one will tend to persist with conservative treatment for a much longer period before subjecting the patient to a second laminectomy. There is also a 2 per cent. risk of developing a further disc prolapse at another level.

\section{Results of Surgery}

Table I gives the results in disc surgery at the hands of experienced surgeons. The figures are an average gathered from several large series.

The overall results of treatment of sciatica or

\section{TABle I}

TABle Showing the Results of Operation for Prolapsed Lumbar INTERvertebral Disc

Completely relieved of sciatic or back pain. .

Greatly improved, mild sciatic or back pain; at full work

Improved, more severe sciatic or back pain; at full or light work

No improvement, or worse; unable to work

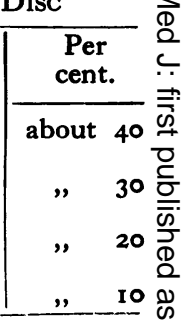

back pain can now be summed up. Out of roo $\vec{\circ}$ patients about 80 will be improved or cured of $\overrightarrow{\vec{\omega}}$ their symptoms by the conservative measures of $\stackrel{\omega}{\sigma}$ bed rest or plaster immobilzation, and 20 will fail 8 to respond. Of these 20 who will eventually come 3 to operation 18 will be made fit to return to work, i and of these eight will be completely relieved of $\dot{\omega}$ symptoms. Two patients will fail to derive any benefit from surgery.

\title{
SUBPHRENIC INFECTION
}

\author{
By A. B. BIRT, F.R.C.S. \\ Norwich
}

The pathology and surgical importance of subphrenic infection cannot be fully understood without a thorough appreciation of the anatomy of the subphrenic space.

\section{Anatomy}

The subphrenic space is defined as being that portion of the abdomen which lies between the diaphragm above and the transverse colon and mesocolon below.

The space is divided into two parts by the liver; the supra-hepatic and infra-hepatic portions.

The supra-hepatic portion is divided into right and left sides by the falciform ligament of the liver. The right side is itself divided into an anterior and posterior space by the right lateral ligament of the liver. The left lateral ligament of the liver runs very close to the posterior margin of the left lobe of the liver so that it does not divide the left suprahepatic area but forms the posterior part of the boundary between the supra- and infra-hepatic parts on the left side. There is, therefore, only one supra-hepatic space on the left side, In addition there is one small extra-peritoneal space in the supra-hepatic part, namely the bare area of the liver, between the leaves of the lateral and falciform ligaments. The supra-hepatic portion of the subphrenic area therefore contains two intra-peritoneal spaces on the right and one on the left and one small extra-peritoneal space. These spaces are usually referred to as the right superior anterior subphrenic space, the right superior posterior subphrenic space, the left superior subphrenic space and the bare area of the liver.

The infra-hepatic portion is divided into right and left parts by the free edge of the lesser $N$ omentum and the descending part of the duodenum. There is only one space on the right in $\mathcal{O}^{-}$ the infra-hepatic region, but on the left there are $\mathrm{W}$ two spaces, an anterior one and a posterior one, being separated from each other by the stomach 0 and the lesser omentum. All the infra-hepatic $\overparen{D}$ spaces are intra-peritoneal, there is no extra- ? peritoneal space below the liver. The infra- 0 hepatic portion of the subphrenic area therefore $\frac{\vec{d}}{\mathbb{D}}$ contains one space on the right and two spaces on 
the left. These spaces are usually referred to as the right inferior subphrenic space, which is the same as the hepatorenocolic pouch of Morrison; the left inferior anterior subphrenic space, often called the perigastric space; and the left inferior posterior subphrenic space, also referred to as the omental bursa or lesser sac of the peritoneum.

From the surgical point of view the space most commonly infected, and therefore the most important, is the right superior posterior space, which is difficult of access both for diagnosis and drainage.

\section{Aetiology}

The commonest cause of subphrenic infection is any infective process occurring in the peritoneal cavity, but less commonly the infection can originate from a more distant focus.

The subphrenic region may be infected by the following methods:

I. Direct extension. This is by direct spread of infection from contiguous organs, such as perforated peptic ulcers and the gall bladder.

2. Distant extension. The commonest cause of infection of the subphrenic region by this means is the appendix or any suppurative lesion in the pelvis. The infection probably spreads mainly by means of retro-peritoneal cellulitis, although retroperitoneal lymphangitis may be the method of spread in some cases. Distant intra-peritoneal foci of infection can also infect the subphrenic region by direct intra-peritoneal drainage of infected material especially if the patient is supine. The diminished incidence of subphrenic infections resulting from the nursing of pelvic infections in Fowler's position probably results from the prevention of this method of spread of infection.

3. Rupture into the subphrenic space. This is the rupture of an adjacent abscess into one of the spaces, such as rupture of a liver abscess.

4. Extension from the thorax. This is not a common source of subphrenic infection, although it certainly does occur. It is probable that the path of spread is by means of retrograde lymphatic extension rather than directly through the diaphragm.

5. Blood stream. In these cases the subphrenic infection is a metastatic infection resulting from septicaemia or pyaemia which has itself resulted from some distant infective focus. This cause accounted for 8 per cent. of a series of 25 cases treated at the Norfolk and Norwich Hospital from 1948 to 1952 .

6. Direct implantation. By this method organisms are implanted directly in the subphrenic region. In wartime this most commonly results from missiles, and in peacetime from aspirating needles inadvertently traversing the diaphragm in the course of the aspiration of an empyema.

In dealing with the actual source of the subphrenic infection most series of cases have shown that about 88 per cent. followed suppuration within the abdomen, about 6 per cent. were bloodborne, about 3 per cent. resulted from extension from the thorax and the remainder were due to a variety of rare causes. Of the causes of abdominal suppuration, most writers report that about 30 per cent. of all subphrenic infections result from the appendix, about 30 per cent. from perforations of the duodenum or the stomach and about 12 per cent. from infections of the liver and biliary passages, the remainder being much less common, from the kidney, pancreas, traumatic, etc.

In a series of cases of subphrenic infection treated at the Norfolk and Norwich Hospital, Norwich, from 1948 to $195^{2}$, no less than 44 per cent. resulted from perforated peptic ulceration, while appendicitis only accounted for 16 per cent. of the cases. It is of interest that, in this series, there were two cases in which subphrenic infection resulted from osteomyelitis of the spine.

The actual infecting organisms vary according to the source of the original infection, but most published figures show that B. coli and streptococos account for almost 40 per cent. of cases each, an $\Phi$ staphylococci for about 20 per cent. of cases. Nof uncommonly the infection is a mixed one.

\section{Diagnosis}

In subphrenic infections the safety and recovery of the patient depends to a great extent on the correct diagnosis being made. The presence of a subphrenic infection can usually be diagnosed if the possibility of its existence is kept well/in mind. In any patient with an unexplained fever, and especially following abdominal suppuration, the likelihood of subphrenic infection should be considered and kept constantly in mind. There is no single test or investigation which will establish the diagnosis. It is only by viewing the case as a whole and keeping the condition constantly in mind that the diagnosis will be arrived at in the early stages and so allow treatment to be started early and thus lower the very high mortality with which this condition has been associated.

Subphrenic infection results in a fever of the swinging type and general toxic signs and symptoms which may result in rapid deterioration in the patient's general condition. The patient may o complain of pain or discomfort in the upper $\mathbb{D}$ abdomen or over the lower ribs posteriorly. $\stackrel{\mathcal{C}}{\rightarrow}$ Tenderness on pressure may be present but its absence by no means excludes the infection. As a rule the tenderness is in relation to the 12 th rib 
posteriorly, or below the costal margins in anterior space infections.

The most useful and constant physical sign is some limitation of movement on respiration, of the lower part of the chest on the affected side. This is usually most easily detected from behind and is due to limitation of diaphragmatic movement on the affected side. Apparent increase in the liver dullness may be noticed. There may be signs of a basal pleural effusion, usually of a sympathetic nature, on the affected side or of basal lobular pulmonary atelectasis. The white blood count, not unnaturally, shows a polymorpho-nuclear leucocytosis. The most useful aid to diagnosis is the use of X-rays, and examination under the fluorescent screen is usually more valuable than by means of films. The most constant X-ray finding is that the diaphragm is raised on the affected side and that the range of movement on respiration is diminished compared with the normal side. It may be possible to see an accumulation of gas in the upper part of the subphrenic abscess with a horizontal fluid level below it, but this is not nearly so common as writers in the past have tended to suggest. The gas is usually due to the fact that the

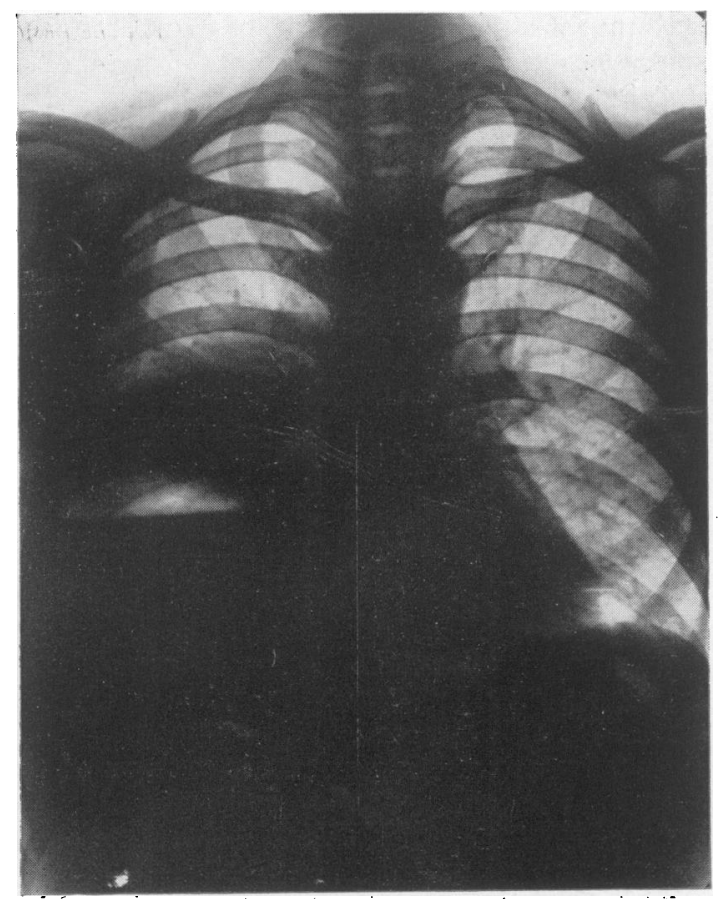

FIG. I.-X-ray film showing a right-sided subphrenic abscess containing gas. The fluid level within the abscess cavity is well shown. Note the raising of the right side of the diaphragm and the degree of right basal pulmonary atelectasis, subphrenic abscess has communicated with a hollow viscus, such as in the case of perforated peptic ulceration, rather than to the presence of gas-forming organisms within the abscess (see Figs. 1, 2 and 3 ).

The X-ray diagnosis of left-sided subphrenic infections may be aided by filling the stomach with barium. This may show the stomach being indented by the abscess at one point with the production of an apparent rounded filling defect of the stomach. Diagnostic pneumo-peritoneum has also been used in the investigation of subphreniinfections. Normally an X-ray after a pneumoc peritoneum has been induced will show the air to have risen and be situated under the diaphragm, whereas in the cases of subphrenic infections adhesions in relation to the area of infection prevent the entry of the air to the sub-diaphragmatic region. Diagnostic aspiration is mentioned only to be condemned, as there is a very great risk that the infection will be spread along the needle track to result in an empyema or spread to other parts of the peritoneum from which it has been successfully walled off by adhesions. Some surgeons advocate aspiration at the time of operation, but the writer does not agree with this view because of the grave risk that the pleura may be traversed.

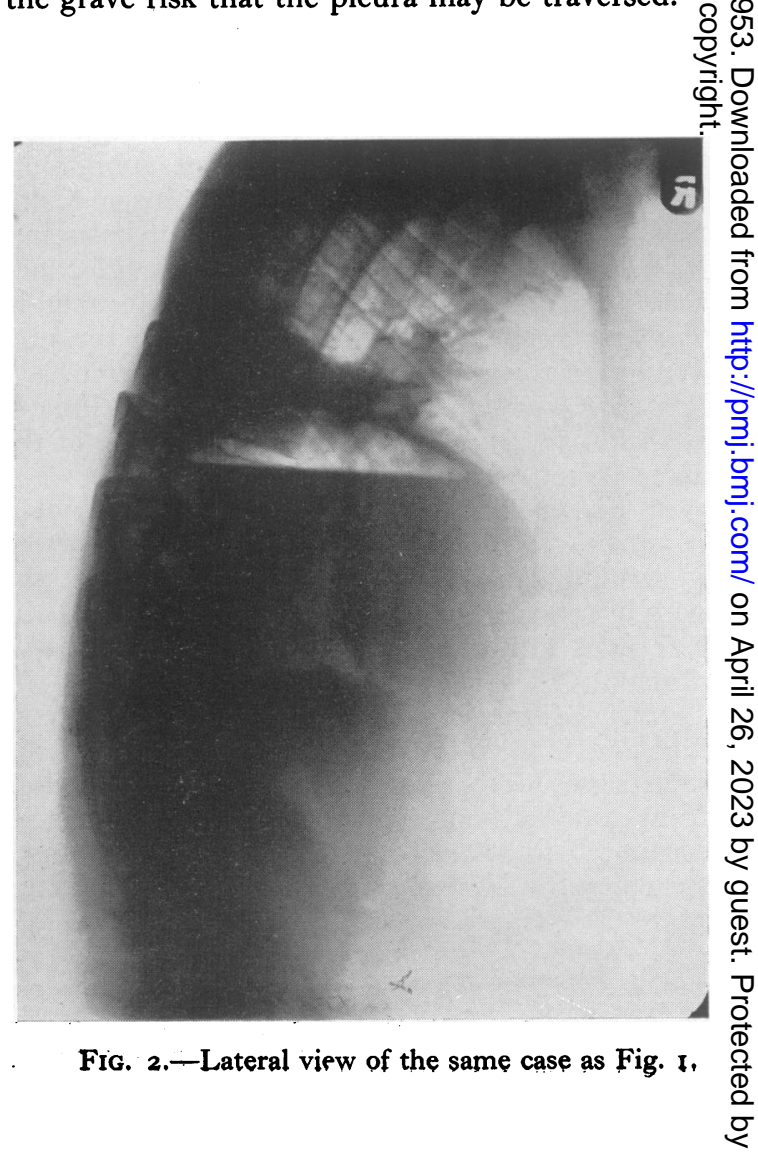




\section{Course of Disease}

As has been stated above, the majority of subphrenic infections are due to some focus of intraabdominal suppuration. Shea and Holden (1948) investigated a large number of cases of subphrenic infection and observed that the average time from the onset of the abdominal suppuration to the suspicion of the presence of subphrenic infection was 15 days.

In considering the course of subphrenic infections it must be realized at the outset that the majority of infections never proceed to suppuration. Those cases which never go on to suppuration remain as a cellulitis of the subphrenic space and then resolve completely, those which do not subside proceed to the formation of an abscess. Ochsner and Graves, in 1933, reported that about 70 per cent. of subphrenic infections subside spontaneously and about 30 per cent. proceed to suppuration. It is important to note this observation which was, of course, made before the days even of sulphonamide therapy. It will, therefore, be appreciated that about two-thirds of all cases of subphrenic infection will recover completely on their own without any form of treatment of the infection.

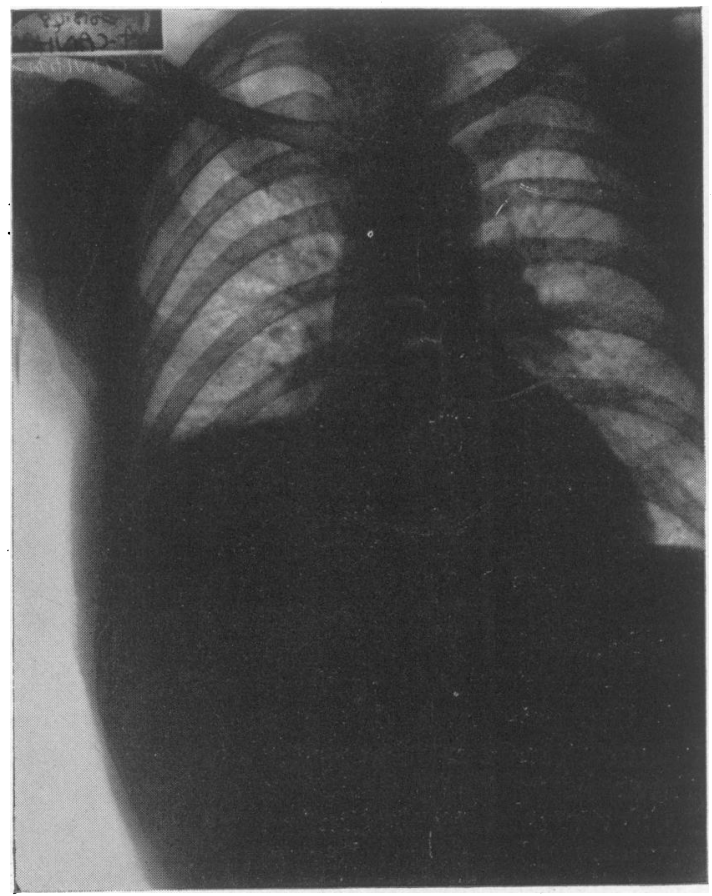

FIG. 3.-X-ray film showing a right-sided subphrenic abscess without any gas and consequently without a fluid level within the abscess cavity.
In considering those cases which have gone on to suppuration it must be stressed at the outset $\frac{2}{z}$ that their prognosis without treatment is poor, and $\stackrel{\otimes}{Q}$ that with energetic and correct treatment the $c$ mortality, can be lowered very greatly. It is $\Rightarrow$ difficult to be certain of the present mortality $\stackrel{\vec{S}}{\rightarrow}$ figures since the advent of antibiotics and chemotherapy, but Ochsner and Graves found that the $\frac{\bar{\sigma}}{\bar{s}}$ mortality in cases of suppuration was as high as $\frac{\bar{C}}{\overrightarrow{0}}$ about 90 per cent. without surgical drainage, and $\stackrel{\varnothing}{\unrhd}$ that with drainage the mortality was lowered to about 33 per cent.

These figures are sufficient evidence to show the $\vec{\circ}$ importance of early diagnosis and correct surgical $\vec{\omega}$ drainage in all cases which have resulted in suppuration.

Although no really accurate figures are available 3 . since the advent of antibiotics, there seems to be तु little doubt that the use of these drugs has pre- $\omega_{j}$ vented as many cases from proceeding to suppura- $\omega_{-}$ tion as was the case formerly, more cases having is undergone resolution in the stage of cellulitis. $\vec{N}$

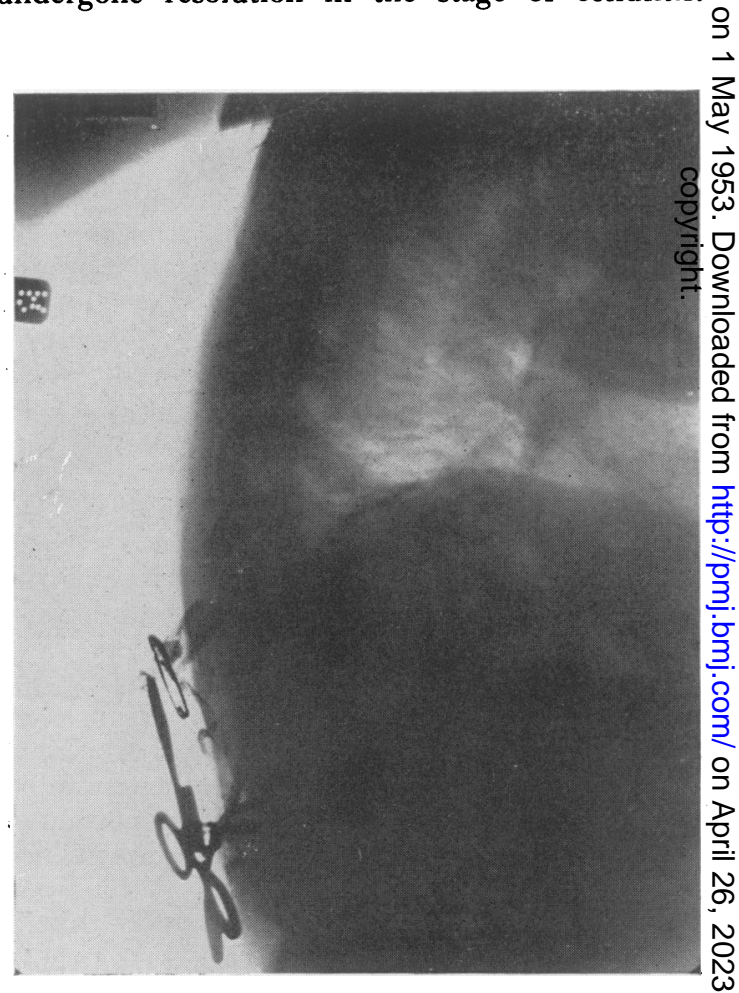

FIG. 4-This lateral view $\mathrm{X}$-ray shows one of the complications of subphrenic abscess. The subphrenic abscess was drained at the site of the lower tube. $\mathbb{\Phi}$ The patient then developed an empyema which was? drained at the site of the upper tube. This film was taken towards the end of treatment after the tubesohad been progressively shortened in length and shortly before their removal. 
Once suppuration has taken place, the importance of early and adequate surgical drainage is just as great as before, but the use of the antibiotics certainly seems to have done a great deal to diminish the intense toxaemia from which these patients suffered, presumably by diminishing the activity of the organisms within the abscess. There is no doubt, clinically, that cases of subphrenic abscess coming to operation today under antibiotic therapy are in a much less toxic state than was the case before the days of antibiotics.

\section{Complications}

The most common complications of subphrenic infections are those affecting the pleura and lung immediately above the part of the diaphragm which is overlying the area of infection. The raising and immobility of the diaphragm results in a high incidence of basal lobular atelectasis of the lung on the affected side, and the atelactatic lung not infrequently becomes infected and results in broncho-pneumonia, which itself may give rise to a lung abscess. Shea and Holden stated that broncho-pneumonia accounted for about 50 per cent. of all the complications resulting from subphrenic infections. Pleural effusions develop not infrequently owing to inflammation of the pleura through the diaphragm. These usually resolve but may go on to give rise to an empyema (Fig. 4). The other complications are comparatively rare and consist mainly of hepatic abscess, septicaemia and pyaemia. In very rare cases a subphrenicpleuro-bronchial fistula may develop, in which case the patient coughs up the pus from the subphrenic abscess.

\section{Treatment}

It cannot be stated too strongly that the mortality of subphrenic suppuration can only be lowered by early diagnosis of the condition, so that the correct treatment can be instituted.

There seems to be little doubt that the institution of antibiotic therapy once the diagnosis of subphrenic cellulitis has been made will prevent at least some cases from proceeding to suppuration and so will aid resolution of a higher proportion of patients. Furthermore, even in those cases which do not resolve, the patient's clinical condition usually remains so much better generally than it does without the use of antibiotics.

The majority of infections are due to streptococci and staphylococci, and penicillin is, therefore, the most useful of the antibiotics for routine use; but it must be remembered that a large number of infections are due to $\mathbf{B}$. coli and to other organisms so that if penicillin produces no response, streptomycin, chloromycetin or aureomycin must be used in addition.
Occasionally the antibiotics will apparently aid resolution of the infection so that the patient seems to be perfectly well and the infection overcome. The administration of the antibiotics is stopped and, after a lapse of time, the infection again manifests itself. In one case, under the writer's care, this actually happened and the patient was discharged from hospital only to return a few weeks later with a return of his pyrexia so that drainage ultimately became necessary. This is due to the fact that all the organisms were not killed in the first place and were therefore able to flare up again, and serves to illustrate the necessity of continuing antibiotic therapy for a sufficiently long time after the infection has apparently been overcome.

Once suppuration has occurred, resolution will only take place in rare cases, and if the high mortality associated with the condition is to be avoided, surgical drainage of the abscess must be undertaken.

Surgical approach of a subphrenic abscess can be either trans-thoracic or trans-abdominal, and in either case the approach can be trans-serous or extra-serous.

Any form of trans-serous approach is to be condemned as this will result in infection of the serous cavity traversed in a high proportion of cases, giving rise to an empyema or peritonitis.

Drainage must, therefore, be carried out by af extra-serous route. Originally this was achieved by the trans-thoracic method, either below the reflection of the pleura or by mobilizing the pleura of the costo-phrenic angle, but in either case there was a grave risk of tearing the pleura and so infecting it. Other methods were tried, firstly suturing the two surfaces of the pleura together, which was not successful as infection spread through the suture holes; and secondly by the two-stage method of preliminary exposure of the parietal pleura and packing down to it so as to promote the formation of adhesions in the pleural cavity and then a few days later draining the abscess through the area of adhesions. This last method was not satisfactory as it meant delaying the actual drainage of the abscess for several days during which time the patient's condition was deteriorating.

The great advance came in 1933 when Ochsner and Graves described the method of transabdominal, extra-serous approach to a subphrenic abscess through the bed of the i2th rib. The right superior posterior subphrenic space is the most commonly affected, and the approach to this space is described. A horizontal incision is made at the level of the outer third of the 12th rib which is then resected periosteally. The dissection is then continued through the outer end of the bed of this 
rib, lateral to the attachment of the arcuate ligament of the diaphragm. The dissection must, therefore, be below the diaphragm and behind the peritoneum. Dissection upwards and forwards in this position must lead to the subphrenic space. This method can also be used on the left side for the approach to a posterior subphrenic abscess on that side.

Anterior subphrenic abscesses are approached by means of subcostal incisions which will lead directly to the abscess cavity extra-peritoneally, as adhesions will have walled off the abscess from the free peritoneal cavity below.

The importance of carrying out drainage by an extra-serous approach is shown by Ochsner and Graves. They found that the mortality of subphrenic abscesses drained by an approach which did not contaminate any serous cavity was about 2 I per cent., while in those drained trans-pleurally the mortality was 39 per cent. and in those drained trans-peritoneally it was 35 per cent.

\section{Summary}

I. The anatomy of the subphrenic region has been described.

2. The aetiology of subphrenic infection has been discussed; most cases follow intra-abdominal suppuration.

3. The diagnosis has been considered. The condition will only be diagnosed early if the surgeon is continually on the look-out for its presence. There is no single method of diagnosis although the radiological findings are of great help.

4. The course of the disease and its main complications have been described. It is to be noted that $\stackrel{0}{\rightarrow}$ about 70 per cent. of cases will resolve on their own without going on to suppuration.

5. The use of antibiotics in the treatment of the $\frac{\bar{D}}{D}$ infection has been described, together with the $\stackrel{\mathbb{Q}}{\Omega}$ methods of drainage of the abscesses. The importance of an extra-serous approach to the abscess is stressed and the method through the bed of the resected 12 th rib is described.

\section{Acknowledgments}

My thanks are due to my colleagues on the 3 . Staff of the Norfolk and Norwich Hospital for iู permission to make use of the notes of cases treated $\dot{\omega}$ by them and for so kindly allowing me to reproduce $\stackrel{\omega}{-}$ some of the X-ray films from their cases.

\section{BIBLIOGRAPHY}

BAILEY, H. (1948), f. Int. Coll. Surg., I1, 377.

FRIEDMAN, P. S. (1950), Radiology, 55, 36.

GROSS, H. T. (1948), Ohio St. Med. F., 44, 1005.

OCHSNER, A., and GRAVES, A. M. (1933), Ann. Surg., 98, 96, $\overrightarrow{0}$

SHEA, P. C., JUN., and HOLDEN, W. D. (1948), Arch. Surg., 58 W 843.

THOREK, P. (1947), Surgery, 21, 739.

\title{
RESUSCITATION OF THE NEWBORN
}

\author{
By Ian Donald, M.B.E., M.D., B.A., M.R.C.O.G. \\ Reader, Institute of Obstetrics and Gynaecology, University of London
}

At no time is a baby likely to be as near death as it is within the first 15 minutes of extra-uterine life. The first few breaths demand a well co-ordinated effort to produce the necessary pressures to open up the lung alveoli. Experimental attempts to inflate collapsed lung artificially usually require pressures of over $40 \mathrm{~cm}$. of water at first. After the lung has once been expanded, lower pressures will succeed in keeping the alveoli open.

For a child to be equal to this effort it must be fit enough to react adequately to the stimuli which the birth process inflicts. The stimuli are of two sorts. Firstly, there are those from skin and joints which crowd in upon the baby's consciousness. Secondly, these are reinforced by the physiological anoxia of delivery. In animals the foetus often falls on to its snout and this provides a very powerful stimulus to respiration.

The carotid sinus, too, plays an important part in respiration as a receptor organ for stimuli, both $\frac{7}{0}$ pressor or chemical, and it is known that breathing is markedly depressed following sinus denervation $N$ in experimental animals. An accumulation of $N$ carbon dioxide will thus stimulate the carotid 0 sinus, but only where it is functionally capable of $\omega$ reacting. Previous anoxia may diminish the response, especially in cases of prematurity and cerebral compression.

\section{Aetiology of Neonatal Asphyxia}

In extreme prematurity, alveolar development $\stackrel{\vec{D}}{\mathrm{D}}$ may not be sufficiently far advanced to sustain 